\title{
Polyaniline Nanofiber Composites with Amines: Novel Materials for Phosgene Detection
}

\author{
Shabnam Virji ${ }^{1}$, Robert Kojima ${ }^{2}$, Jesse D. Fowler ${ }^{1}$, Joey G. Villanueva ${ }^{2}$, Richard B. Kaner ${ }^{2}$, and Bruce H. \\ Weiller $^{1}(\bowtie)$ \\ ${ }^{1}$ Materials Processing and Evaluation Department, Space Materials Laboratory, The Aerospace Corporation, P. O. Box 92957/M2-248, \\ Los Angeles, CA 90009, USA \\ 2 Department of Chemistry \& Biochemistry and California NanoSystems Institute, University of California, Los Angeles, Los Angeles, \\ CA 90095-1569, USA \\ Received: 29 August 2008 / Revised: 9 December 2008 / Accepted: 10 December 2008 \\ CTsinghua University Press and Springer-Verlag 2009. This article is published with open access at Springerlink.com
}

\begin{abstract}
Several orders of magnitude of change in resistance are observed upon chemical doping and dedoping of the conducting polymer polyaniline. This large conductivity range can be utilized to make sensitive chemical sensors. Polyaniline, in its nanofiber form, has even greater sensing capabilities due to the small fiber diameters, high surface area, and porous nanofiber network that enhances gas diffusion into the fibers. Polyaniline nanofibers have been synthesized using a rapid mixing method and dispersed in water allowing them to be easily modified with water soluble agents, making new composite materials. Polyaniline nanofiber composite materials can be used to enhance detection of analytes that unmodified polyaniline would not otherwise be able to detect. The detection mechanism involves the reaction of an additive with the analyte to generate a strong acid that is easily detected by polyaniline, resulting in orders of magnitude changes in resistance. The reaction of the additive alone with the analyte produces no electrical response, however. In this paper, an array of amine-polyaniline nanofiber composite materials is investigated for the detection of phosgene gas. The influence of environmental conditions such as humidity and temperature are examined and a detection mechanism is presented.
\end{abstract}

\section{KEYWORDS}

Polyaniline nanofibers, conducting polymer, amines, chemical sensor, phosgene

\section{Introduction}

Polyaniline is a widely used conducting polymer for many applications including chemical sensors [1], anti-corrosion coatings [2], light emitting diodes [3], actuators [4], and electrochromic windows [5] due to its wide range in conductivity resulting from chemical doping and dedoping. The oxidation state of polyaniline can be reversibly changed from the insulating emeraldine base form $\left(\sigma<10^{-10} \mathrm{~S} / \mathrm{cm}\right)$ to the conducting emeraldine salt form $(\sigma>1 \mathrm{~S} / \mathrm{cm})$ by simple acid and base doping and dedoping [6]. Here, this large conductivity change is utilized to develop polyaniline sensors to detect phosgene, a toxic

Address correspondence to bruce.h.weiller@aero.org 
industrial chemical.

Polyaniline nanofibers possess all the useful properties of conventional polyaniline with the added advantage of having a high surface area due to their small fiber diameters $(\sim 50 \mathrm{~nm})$ that allows for better gas diffusion leading to enhanced sensor response [1]. Polyaniline nanofibers can be readily obtained using several synthetic approaches including a rapid mixing process [7], interfacial polymerization [8], seeded growth [9, 10], dilute polymerization [11], use of structure directing agents [12], templated growth [13] and use of new oxidizers [14, 15]. Here we use the rapid mixing method, so that pure nanofibers are obtained that do not need to be separated from a substrate, structure directing agent or surfactant. This method produces polyaniline nanofibers that readily disperse in water. The nanofiber diameter can also be tuned from $30-120 \mathrm{~nm}$ depending on the acid used in the synthesis.

Since water is used as the solvent to disperse the polyaniline nanofibers, different water soluble agents can be used to create new composite materials. We have previously shown that metal salt modified polyaniline nanofibers detect hydrogen sulfide with orders of magnitude change in resistance [16] but the unmodified polyaniline nanofibers do not. The metal salt modified polyaniline nanofibers react with hydrogen sulfide to generate a metal sulfide and a strong acid that in turn dopes the polyaniline nanofibers resulting in large conductivity changes. In addition to inorganic salts as additives for hydrogen sulfide sensors, we have also shown that organic additives such as hexafluoroisopropanol can be used for hydrazine sensing. This reaction utilizes a unique chemical reaction of hexafluoroisopropanol with hydrazine to generate hydrofluoric acid (HF) that dopes polyaniline, resulting in orders of magnitude changes in resistance [17]. Using this strategy, new composite materials can be synthesized to detect other toxic chemicals such as phosgene.

Phosgene $\left(\mathrm{COCl}_{2}\right)$ is a highly toxic industrial chemical that has a low permissible exposure limit of $0.1 \mathrm{ppm}$ [18]. It is currently used in industry to make plastics and pesticides and was used as a chemical weapon during World War I. Phosgene is colorless, exhibits the odor of freshly cut hay or green corn that is not easily noticed, and exposure causes severe lung injuries [18]. Various methods have been examined to detect phosgene including spectroscopic [19] and optical techniques [20]. In this paper, polyaniline nanofiber-amine composite materials are used to detect phosgene. These sensors operate at room temperature, are easy to fabricate, and detect phosgene with over two orders of magnitude change in resistance. The sensing mechanism, as well as the effects of environmental factors such as humidity and temperature are presented and discussed.

\section{Experimental}

All reagents except $\mathrm{NaOH}$ and ammonium peroxydisulfate (APS) were purchased from Aldrich. $\mathrm{NaOH}$ and APS were purchased from Fisher Scientific. All chemicals were of ACS grade and used as received except for aniline which was distilled prior to use.

Polyaniline nanofibers were synthesized using a rapid mixing method [7]. A typical synthesis consists of combining $10.0 \mathrm{~mL}$ of an aqueous camphorsulfonic acid (CSA) solution (1.0 mol/L) containing $0.200 \mathrm{~mL}$ of aniline $(2.20 \mathrm{mmol})$ with $10.0 \mathrm{~mL}$ of an aqueous CSA solution $(1.0 \mathrm{~mol} / \mathrm{L})$ containing $0.125 \mathrm{~g}$ of APS ( $0.55 \mathrm{mmol})$. The two solutions were rapidly mixed and then allowed to react overnight. The resulting emeraldine salt polyaniline nanofibers were then dedoped with an equal volume of aqueous $1 \mathrm{~mol} / \mathrm{L}$ $\mathrm{NaOH}$ and purified by centrifugation. The resulting nanofiber suspension in water was diluted to obtain a final concentration of $2 \mathrm{~g} / \mathrm{L}$.

Using water as the solvent for the nanofiber suspension allows easy modification of the polyaniline nanofibers with water soluble agents. In particular, water soluble amines can be used to modify polyaniline to allow detection of analytes that unmodified polyaniline cannot detect. A typical synthesis of such a composite material involves addition of a $0.005 \mathrm{~mol} / \mathrm{L}$ solution of a water soluble amine to $1.6 \mathrm{~g} / \mathrm{L}$ polyaniline nanofibers suspended in water. The amines used include ethylenediamine, phenylenediamine and metanilic acid (3-aminobenzenesulfonic acid), and the amine salts phenylenediamine dihydrochloride and 


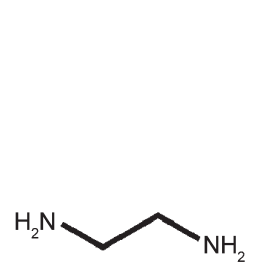

Ethylenediamine

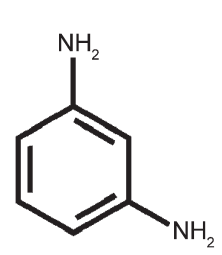

Phenylenediamine

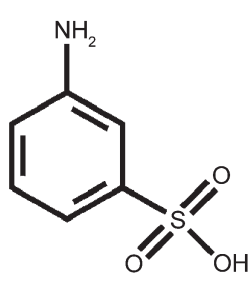

Metanilic acid
Scheme 1 Structures of the amines used

ethylenediamine dihydrochloride (Scheme I). Films of these composite materials were made by drop-casting using disposable microliter pipettes and drying in air.

The sensor array substrate contains 18 sensors with each sensor having 35 pairs of fingers with $20 \mu \mathrm{m}$ electrode gaps. Two of these packaged sensor arrays sit in the cell, giving 36 sensors in total. The sensor arrays were fabricated in house using standard photolithography. Nitrogen was used as the buffer gas. Mass-flow controllers were used to meter separate flows of nitrogen buffer gas and the calibrated gas mixture. The total flow rate remained constant throughout each experiment with a nitrogen buffer balance flow when the valve was off and no analyte gas was flowing. A permeation tube of phosgene (KinTek) was used to generate phosgene gas. The gas concentrations were verified using Dräger tubes. A bubbler filled with water and a humidity sensor (Visala) were used to generate and measure humidity. In order to keep the toxic gas confined and the experiment safe, all measurements were performed in a fume hood with a scrubber in place to scavenge all exhaust gases.

A programmable electrometer (Keithley 2002) was used to measure electrical resistance. A scanner card and switch system (Keithley 7011S/7001) was used to multiplex measurements over the 36 sensors in the two packaged sensor elements. All instruments were controlled and read by computer using a GPIB interface and Labview software.

Scanning electron microscope images were taken on a JEOL, model JSM-6460LV scanning electron microscope. A Nicolet Magna 550-IR spectrometer with a Nic-Plan IR microscope and a mercury cadmium telluride detector was used to obtain the Fourier transform infrared (FT-IR) spectra.

\section{Results and discussion}

Figure 1 shows the scanning electron microscope image of the polyaniline nanofibers cast on sensor electrode substrates. As can be seen from the figure, the films are completely nanofibrous, with fiber diameters ranging from 30 to $50 \mathrm{~nm}$, and fiber lengths varying from $500 \mathrm{~nm}$ to a few microns. The porosity and high surface area of the nanofibers are evident and enable enhanced sensor performance due to better gas diffusion than that observed with conventional polyaniline [21, 22].

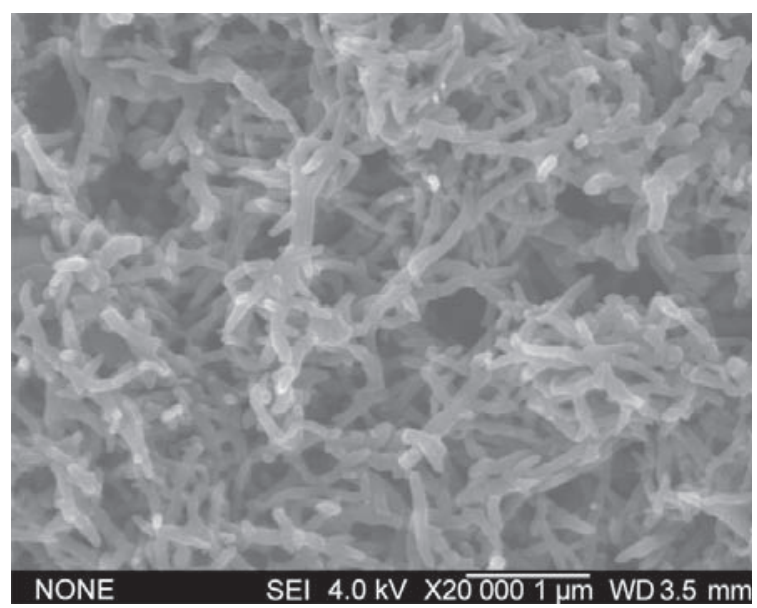

Figure 1 Scanning electron microscope image of polyaniline nanofibers

Phosgene has a permissible exposure limit (PEL) of $0.1 \mathrm{ppm}$ and an immediate danger to health and life (IDLH) limit of 2 ppm [18]. Unmodified polyaniline cannot detect phosgene at these low exposure concentrations. We have previously shown that metal salt-polyaniline nanofiber composite materials can detect gases such as hydrogen sulfide using chemical reactions that generate a strong acid [16]. However, there are no known metal salt reactions with acid chlorides that generate any acidic byproduct that can react with polyaniline. Therefore, another additive is needed that reacts with phosgene to produce a product that can dope the polyaniline nanofibers.

We have found that composites of polyaniline nanofibers with amines respond well to phosgene at concentrations lower than $0.01 \mathrm{ppm}$. Figure 2 shows the response curves of different polyaniline nanofiber composite films to phosgene. The 


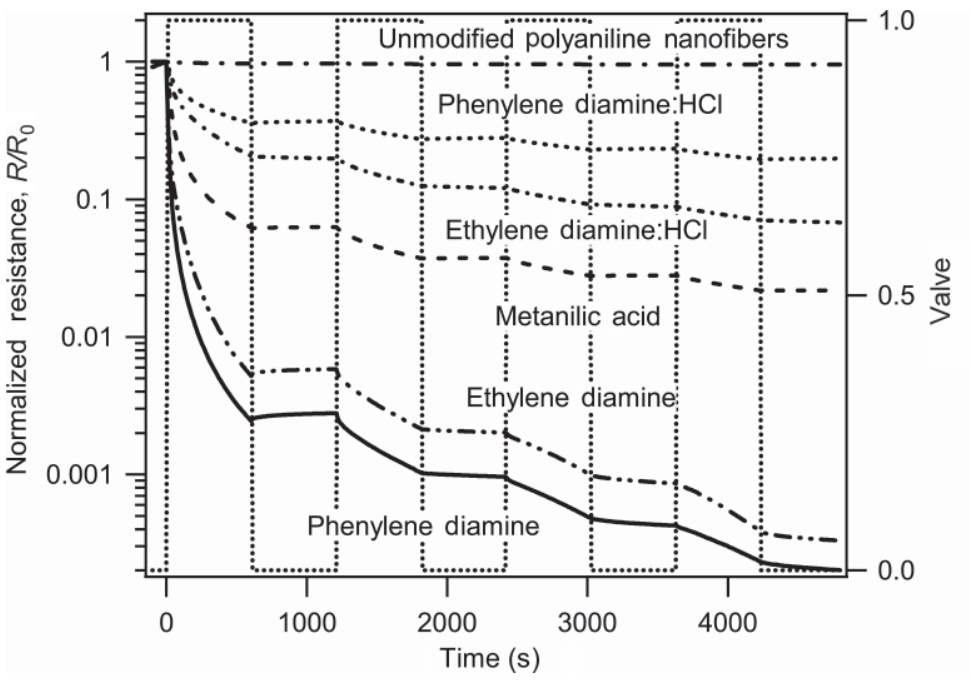

Figure 2 Response of polyaniline nanofiber amine composites to phosgene. The concentration of phosgene is $2 \mathrm{ppm}$ at room temperature with $50 \%$ relative humidity. The amines used are phenylene diamine dihydrochloride $(\cdots \cdots)$, ethylene

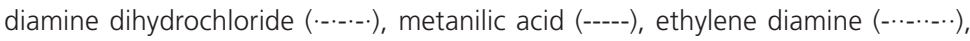
and phenylene diamine (-). Also, shown is the response of an unmodified polyaniline nanofiber film (- - - -). The valve state is shown on the right axis
There is a large difference in response to phosgene depending on the particular amine used. Here both the neat amine and amine salts were used. The response is dramatically different for the neat amines compared to the amine salts. The amine-polyaniline nanofiber composite materials are synthesized in aqueous solution by addition of the amine solution to an aqueous suspension of polyaniline nanofibers. When the amine salts are dissolved in water they dissociate to create an acidic solution together with free amines. The addition of this slightly acidic solution to the aqueous polyaniline nanofiber suspension partially dopes the polyaniline nanofibers. This partial doping results in a decrease in the initial resistance and a smaller dynamic range over which the polyaniline nanofibers can respond to the fully doped amines and amine salts used are ethylenediamine, ethylenediamine dihydrochloride, phenylenediamine, phenylenediamine dihydrochloride, and metanilic acid (Scheme 1). The left axis is the normalized resistance which is the resistance at a given time divided by the initial resistance, the $x$-axis is the time, and the right axis shows the valve state. As seen in the figure, different amine-polyaniline nanofiber composite materials interact with phosgene differently. In each case there is a doping response suggesting formation of an acid product, similar to the response of the metal salt-polyaniline nanofiber films to hydrogen sulfide [16].

Amines are known to react with phosgene in a nucleophilic substitution reaction to form carbamoyl chlorides which can be readily dehydrohalogenated to form isocyanates (Scheme 2) [23]. In this reaction hydrochloric acid is formed, which can dope the polyaniline converting it from the emeraldine base oxidation state to the emeraldine salt oxidation state. This results in two orders of magnitude decrease in resistance (Fig. 2).

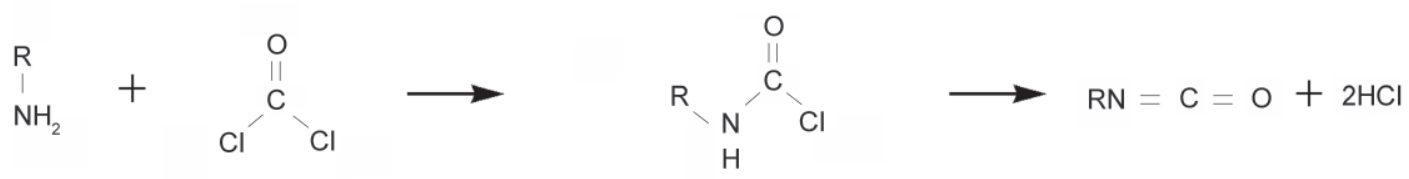

Scheme 2 Reaction of phosgene with amines state. As a result, the response of the amine salt composite materials is much smaller than that for the neat amine composites as can be seen in Fig. 2 for ethylenediamine and phenylenediamine compared with their dihydrochloride salts.

The difference in the response levels for the different amines may also be due to their different acid dissociation constants $\left(p K_{\mathrm{a}}\right)$ that affects their interaction with the polyaniline nanofibers. The $\mathrm{p} K_{\mathrm{a}}$ values for ethylenediamine, phenylenediamine, and metanilic acid are 9.92, 5.11, and 3.74, respectively [24]. Polyaniline is known to be doped by acids that have $\mathrm{p} K_{\mathrm{a}}$ values below 4.75 [25]. Therefore, metanilic acid is able to partially dope polyaniline in the same way as the dihydrochloride salts of ethylenediamine and phenylenediamine. This partial doping decreases the initial resistance and results in a smaller resistance range over which the polyaniline nanofibers can be fully doped and these composites have the lowest response to phosgene. Both phenylenediamine and ethylenediamine have $\mathrm{p} K_{\mathrm{a}}$ values $>4.75$ and will not dope polyaniline; these composites have the greatest 
response to phosgene.

In addition to affecting the doping level of polyaniline, the different $\mathrm{p} K_{\mathrm{a}}$ values give the amines different nucleophilicities. Phosgene undergoes a nucleophilic substitution reaction with amines as indicated in Scheme 1 which is affected by the nucleophilicity of the amines. The more basic the amine (i.e., the higher the $\mathrm{p} K_{\mathrm{a}}$ ), the more reactive it is as a nucleophile. As the amine becomes more nucleophilic, its rate of reaction with phosgene (Scheme 2) increases. This is seen in Fig. 2 when comparing the response of the ethylenediamine composites and the phenylenediamine composites. For the dihydrochloride salts, the ethylenediamine composites have a greater response than the phenylenediamine composites. Although the composite with free ethylenediamine can be used to detect phosgene, since ethylenediamine is a liquid it is likely to vaporize and the amount present in the film can change over time causing a variable response to phosgene. Although the composite with ethylenediamine can be used to detect phosgene, since it is a liquid it is more likely to vaporize and the amount present in the film can change over time affecting its response to phosgene.

As shown in Fig. 3, these materials are able to detect phosgene well below the PEL of $0.1 \mathrm{ppm}$. Even at the PEL level, the phenylenediamine-polyaniline nanofiber composite material can detect phosgene with orders of magnitude change in resistance.

The influence of environmental factors such as humidity and temperature on the response of the amine composites with polyaniline nanofibers was also studied. Figure 4 shows the response of the phenylenediamine and phenylenediamine dihydrochloride composite films to phosgene at room temperature under varying relative humidity levels. Moisture is known to increase the conductivity of polyaniline due to enhanced charge transfer along the polyaniline chain [26]. As a result, in the presence of a humid environment the polyaniline nanofibers become more conducting, leading to enhanced charge propagation along the chain upon acid doping. As the relative humidity increases, the response to phosgene improves as can be seen in Fig. 4. Similar effects are observed in simple acid doping of polyaniline nanofibers. Note that the scale for the hydrochloride salt in Fig. 4 is greatly expanded and gives the impression of larger baseline change at $0 \%$ than for the free amine. As the humidity decreases, the response
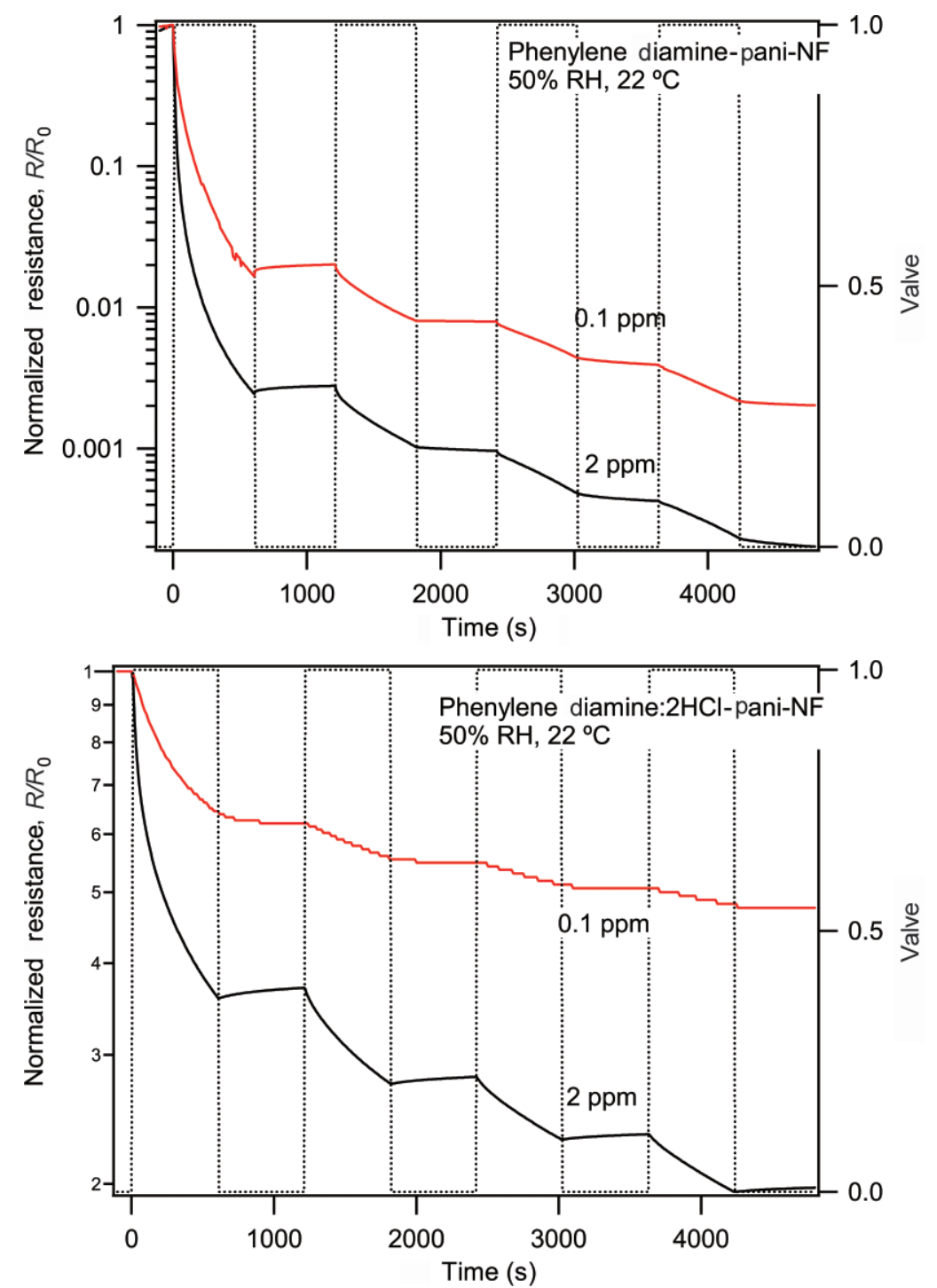

Figure 3 Concentration dependence of the response of polyaniline nanofiber amine composite materials to phosgene. The amines used were phenylene diamine and phenylene diamine dihydrochloride, the phosgene concentrations were 0.1 and 2 ppm phosgene at room temperature with $50 \%$ relative humidity. The valve state (dashed line) is show on the right axis 


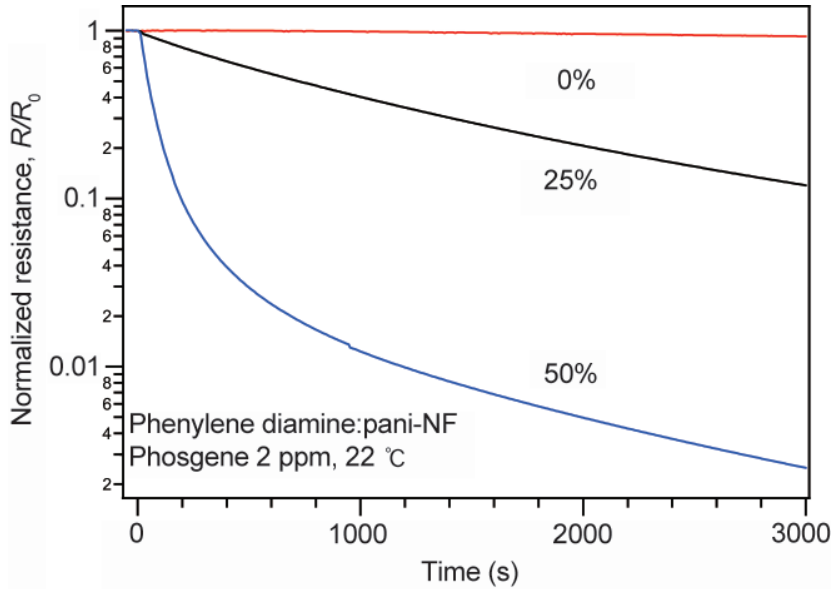

(a)

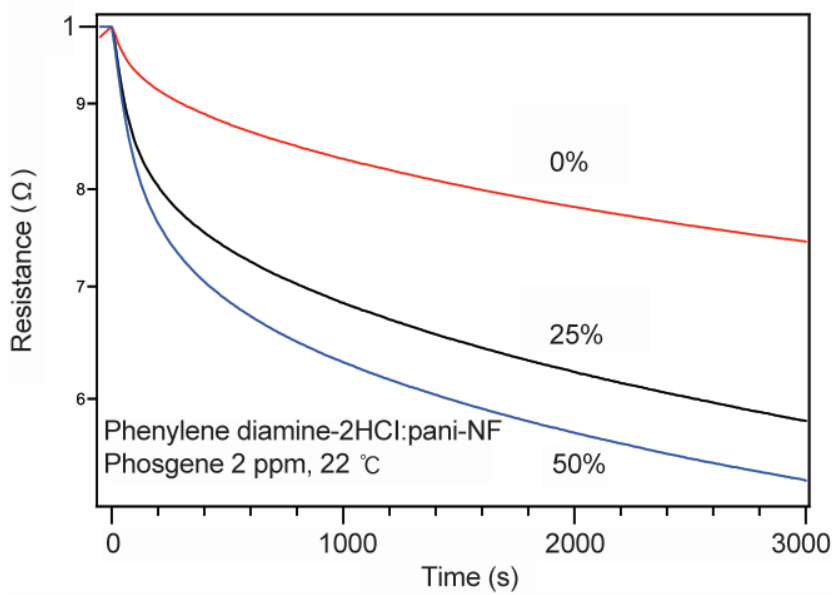

(b)

Figure 4 Humidity dependence of the response of polyaniline nanofiber amine composites to phosgene. The amines used are phenylene diamine (a) and phenylene diamine dihydrochloride (b) exposed to $2 \mathrm{ppm}$ phosgene at room temperature with $0 \%, 25 \%$, and $50 \%$ relative humidity. In this case the exposure is continuous. Note that the scale for the lower figure is greatly expanded

is inhibited, as can also be seen with the decrease in response at increased temperature (Fig. 5). In these experiments the absolute water vapor concentration was held constant so as the temperature increases, the amount of water in the film decreases, and therefore charge propagation along the fibers decreases.

In order to further examine the reaction mechanism, the FT-IR spectra of an ethylenediaminepolyaniline nanofiber composite material were examined before and after exposure to phosgene (Fig. 6). The FT-IR spectra were obtained from reflectance measurements of the films directly on the sensor electrodes by using an IR microscope. The polyaniline nanofiber composite material was dropcast onto the sensor, air dried and its reflectance IR

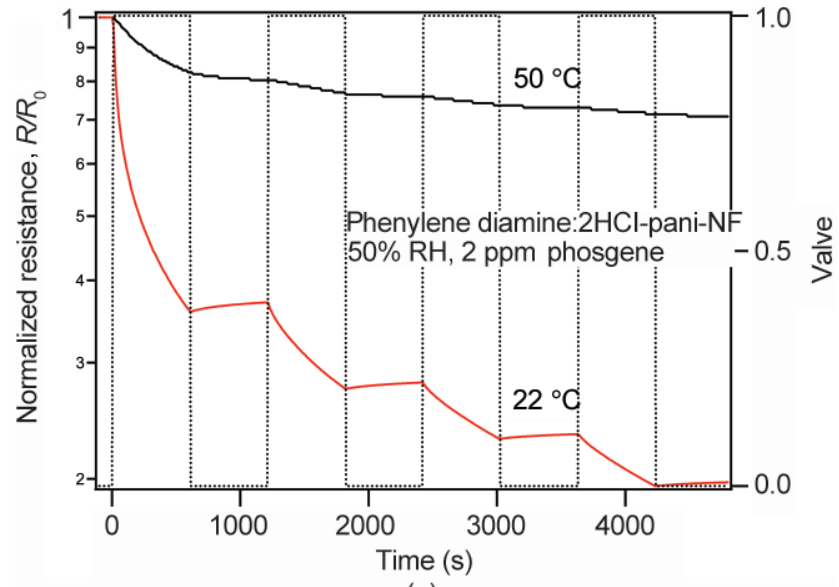

(a)

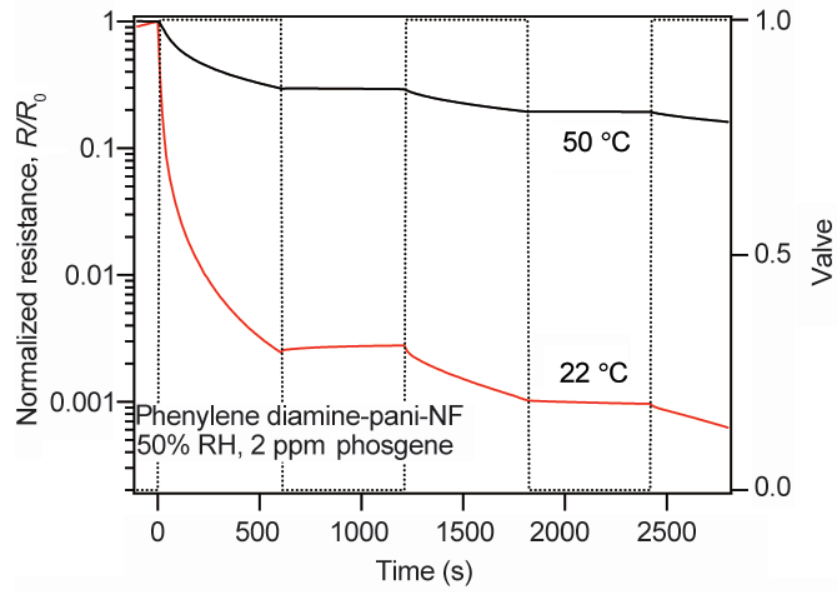

(b)

Figure 5 Temperature dependence of the response of polyaniline nanofiber amine composites to phosgene. The amines used were phenylene diamine and phenylene diamine dihydrochloride and the concentration was $2 \mathrm{ppm}$ phosgene at $22{ }^{\circ} \mathrm{C}$ and $50{ }^{\circ} \mathrm{C}$ with $50 \%$ relative humidity. The valve state (dashed line) is shown on the right axis

spectrum was recorded. The film was then exposed to phosgene and the spectrum after exposure was recorded using the same method. Table 1 shows the FT-IR peak assignments for the emeraldine base and emeraldine salt oxidation state of polyaniline [17], neat ethylenediamine [27], gaseous phosgene [28], and the ethylenediamine-polyaniline nanofiber composite material before and after phosgene exposure. As shown in Table 1, there is a new peak that forms at $1741 \mathrm{~cm}^{-1}$ indicative of a carbonyl. This new peak is shifted from the $\mathrm{C}=\mathrm{O}$ stretch of phosgene at $1820 \mathrm{~cm}^{-1}$ suggesting that a new compound with a $\mathrm{C}=\mathrm{O}$ functional group is formed after exposure to phosgene. The nucleophilic substitution reaction proposed in Scheme 2 assumes 


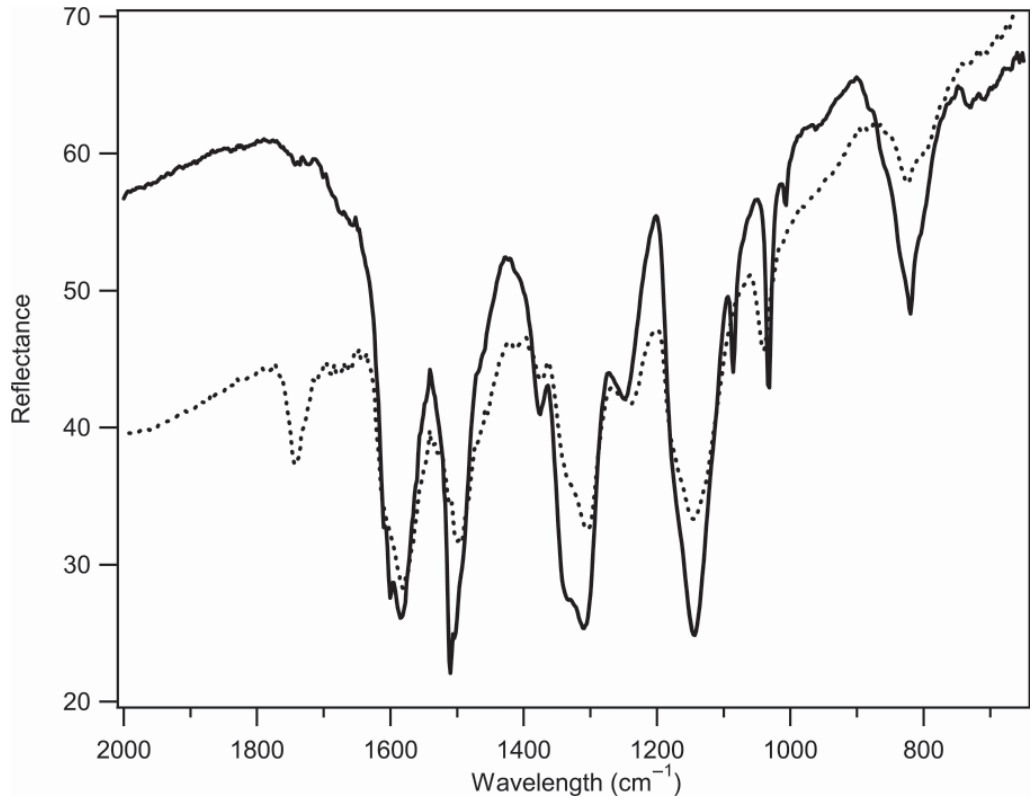

Figure 6 FT-IR spectra of polyaniline nanofiber composite films with ethylenediamine before (-) and after (---) exposure to 2 ppm phosgene at room temperature and 50\% relative humidity. The appearance of the carbonyl peak at $1741 \mathrm{~cm}^{-1}$ is indicative of the reaction of phosgene with the amine in the film as in Scheme 2

Table 1 Infrared peak assignments for polyaniline nanofiber amine composite film before and after reactions with phosgene

\begin{tabular}{c|c|c|c}
\hline Peak assignments & Phosgene & $\begin{array}{c}\text { Ethylenediamine-composite } \\
\text { (Before phosgene) }\end{array}$ & $\begin{array}{c}\text { Ethylenediamine-composite } \\
\text { (After phosgene) }\end{array}$ \\
\hline Quinoid ring stretch & & 1583 & 1581 \\
\hline Quinoid ring vibration & & 1510 & 1496 \\
\hline Benzenoid ring C-N stretch & & 1311 & 1303 \\
\hline Quinoid ring C-N stretch & & 1145 & 1145 \\
\hline Quinoid ring C-H bend & & 819 & 1041 \\
\hline $\mathrm{C}-\mathrm{H}$ stretch & & 1031 & \\
\hline $\mathrm{CH}_{2}$ and $\mathrm{NH}_{2}$ vibration & $1682,856-848$ & & 1741 \\
\hline $\mathrm{C}-\mathrm{Cl}$ & $1832-1820$ & &
\end{tabular}

a Peak assignments are given in wavenumbers $\left(\mathrm{cm}^{-1}\right)$.

that a carbamoyl chloride is formed and reacts further to form an isocyanate both of which have a $\mathrm{C}=\mathrm{O}$ group. A new carbonyl peak is found in the FT-IR spectrum after phosgene exposure (Fig. 6), suggesting that this reaction is occurring and is responsible for the sensor response.

\section{Conclusions}

Phosgene can be detected well below the permissible exposure limit $(0.1 \mathrm{ppm})$ with amine modified polyaniline nanofiber composite materials. The reaction mechanism is based on a nucleophilic substitution reaction of phosgene with the amine to generate an isocyanate and an acid. The acid dopes the polyaniline nanofibers increasing their conductivity by up to two orders of magnitude. Various amines and amine chlorides have been examined and the response is found to be dependent on several factors including the acidity of the amine and the doping level of the polyaniline. The amine chlorides, when dissolved in water, release acid and have the ability to partially dope polyaniline resulting in a decrease in resistance. The acidity of the neat amine also affects its nucleophilicity. The more basic the amine, the more reactive it is as a nucleophile. Here, phenylenediamine is more basic than metanilic acid and enhances the nucleophilic substitution reaction resulting in a larger response to phosgene. Humidity and temperature affect the response of the composite materials to phosgene. Humidity enhances charge propagation along the polymer chain and increases the response of the film to phosgene. Temperature, on the other hand, reduces the response to phosgene because on heating the amount of water vapor in the film decreases. However, these inexpensive sensors are irreversible dosimeters for phosgene and as such are best suited to single use applications.

\section{Acknowledgements}

This work was supported by the Aerospace Corporation's Independent Research and Development Program (BHW) and the National Science Foundation Grant DMR 0507294 (RBK). 


\section{References}

[1] Huang, J.; Virji, S.; Weiller, B. H.; Kaner, R. B. Polyaniline nanofibers: Facile synthesis and chemical sensors. J. Am. Chem. Soc. 2003, 125, 314-315.

[2] Chaudhari, S.; Patil, P. P. Corrosion protective bi-layered composites of polyaniline and poly(o-anisidine) on low carbon steel. J. Appl. Polym. Sci. 2008, 109, 2546-2561.

[3] Yang, Y.; Westerweele, E.; Zhang, C.; Smith, P.; Heeter, A. Enhanced performance of polymer light-emitting-diodes using high-surface-area polyaniline network electrodes. J. Appl. Phys. 1995, 77, 694-698.

[4] Baker, C. O.; Shedd, B.; Innis, P. C.; Whitten, P. G. Spinks, G. M.; Wallace, G. G.; Kaner, R. B. Monolithic actuators from flash-welded polyaniline nanofibers. Adv. Mater. 2008, 20, 155-158.

[5] Jelle, B. P.; Hagen, G. Electrochemical multilayer deposition of polyaniline and Prussian blue and their application in solid state electrochromic windows. J. Appl. Elctrochem. 1998, 28, 1061-1065.

[6] Huang, W. -S.; Humphrey, B. D.; MacDiarmid, A. G. Polyaniline, a novel conducting polymer. J. Chem. Soc, Faraday Trans. 1986, 82, 2385-2400.

[7] Huang, J.; Kaner, R. B. Nanofiber formation in the chemical polymerization of aniline: A mechanistic study. Angew. Chem. Int. Ed. 2004, 43, 5817-5821.

[8] Huang, J.; Kaner, R. B. A general chemical route to polyaniline nanofibers. J. Am. Chem. Soc. 2004, 126, 851-855.

[9] Zhang, X.; Goux, W.; Manohar, S. K. Synthesis of polyaniline nanofibers by "nanofiber seeding". J. Am. Chem. Soc. 2004, 126, 4502-4503.

[10] Xing, S.; Zhao, C.; Jing, S.; Wang, Z. Morphology and conductivity of polyaniline nanofibers prepared by "seeding" polymerization. Polymer 2006, 47, 23052313.

[11] Chiou, N.; Epstein, A. J. Polyaniline nanofibers prepared by dilute polymerization. Adv. Mater. 2005, 17, 16791683.

[12] Zhang, X.; Manohar, S. K. Polyaniline nanofibers: Chemical synthesis using surfactants. Chem. Commun. 2004, 20, 2360-2361.

[13] Li, X.; Zhang, X.; Li, H. Preparation and characterization of pyrrole/aniline copolymer nanofibrils using the template-synthesis method. J. Appl. Polym. Sci. 2001, 81, 3002-3007.

[14] Rahy, A.; Yang; D. J. Synthesis of highly conductive polyaniline nanofibers. Mater. Lett. 2008, 62, 43114314.

[15] Rahy, A.; Sakrout, M.; Manohar, S.; Cho, S. J.; Ferraris, J.; Yang, D. J. Polyaniline nanofiber synthesis by co-use of ammonium peroxydisulfate and sodium hypochlorite. Chem. Mater. 2008, 20, 4808-4814.

[16] Virji, S.; Fowler, J. D.; Baker, C. O.; Huang, J.; Kaner, R. B.; Weiller, B. H. Polyaniline manofiber composites with metal salts: Chemical sensors for hydrogen sulfide. Small 2005, 1, 624-627.

[17] Virji, S.; Kaner, R. B.; Weiller, B. H. Hydrazine detection by polyaniline using fluorinated alcohol additives. Chem. Mater. 2005 17, 1256-1260.

[18] Centers for Disease Control and Prevention. http://www. bt.cdc.gov/agent/phosgene/basics/facts.asp (accessed Aug. 29, 2008).

[19] Hill, H. H.; Martin, S. J. Conventional analytical methods for chemical warfare agents. Pure Appl. Chem. 2002 74, 2281-2291.

[20] Frye-Mason, G.; Leuschen, M.; Wald, L.; Paul, K.; Hancock, L. F. Reactive chromophores for sensitive and selective detection of chemical warfare agents and toxic industrial chemicals. Proc. SPIE 2005, 5778, 337-346.

[21] Virji, S.; Huang, J.; Kaner, R. B.; Weiller, B. H. Polyaniline nanofiber gas sensors: Examination of response mechanisms. Nano Lett. 2004, 4, 491-496.

[22] Huang, J.; Virji, S.; Weiller, B. H.; Kaner, R. B. Nanostructured polyaniline sensors. Chem. Eur. J. 2004, 10, 1315-1319.

[23] Babad, H.; Zeiler, A. G. Chemistry of phosgene. Chem. Rev. 1973, 73, 75-91.

[24] CRC Handbook of Chemistry and Physics, 88 edition [Online]. http://www.hbcpnetbase.com (accessed Aug 29, 2008).

[25] Hatchett, D. W.; Josowicz, M.; Janata, J. Acid doping of polyaniline: Spectroscopic and electrochemical studies. J. Phys. Chem. B. 1999, 103, 10992-10998.

[26] Javadi, H. H. S.; Angelopoulos, M.; MacDiarmid, A. G.; Epstein, A. J. Conduction mechanism of polyanilineeffect of moisture. Synthetic Met. 1988, 26, 1-8.

[27] Segal, L.; Eggerton, F. V. Infrared spectra of ethylenediamine and the dimethylethylenediamines. Appl. Spec. 1961, 15, 116-117.

[28] Joung, S. -L.; Amemiya, T.; Murabayashi, M.; Cai, R.; Itoh, K. Chemical adsorption of phosgene on $\mathrm{TiO}_{2}$ and its effect on the photocatalytic oxidation of trichloroethylene. Surf. Sci. 2005, 598, 174-184. 